\title{
DKG
}

KREBSGESELLSCHAFT

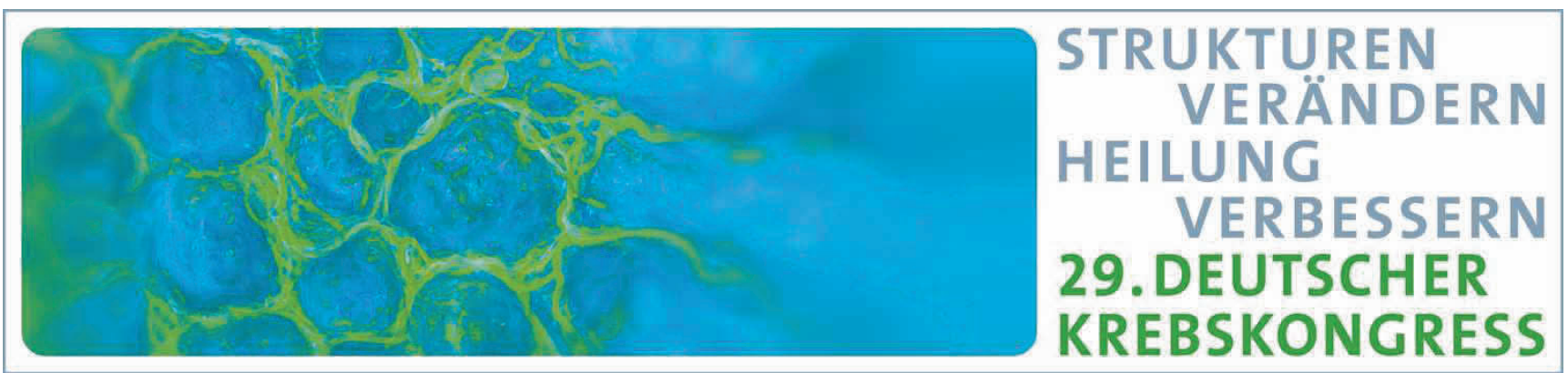

Neue sogenannte "targeted therapies" und erste etablierte Biomarker zur Vorhersage eines Therapieerfolgs haben dazu beigetragen, dem Ziel einer personalisierten Tumortherapie näher zu kommen. Personalisierte Tumortherapie, das heißt, Patienten mit Tumorerkrankungen individuell eine wirksame und sichere Therapie anbieten zu können. Es bedeutet demzufolge auch, ineffektive Therapien zu vermeiden und Kosten zu senken. Die bestmögliche und zugleich effiziente Versorgung der Tumorpatienten steht auch im Mittelpunkt der aktuellen Bestrebungen zur Verbesserung von Versorgungsstrukturen durch beispielsweise Therapieleitlinien, Zentrumsbildung und sektorenübergreifende Therapiekonzepte im
Rahmen des Nationalen Krebsplans. Wichtige Aspekte dabei sind die Förderung der interdisziplinären Zusammenarbeit aller an der Tumortherapie beteiligten Fachgruppen und Institutionen und der effiziente Einsatz der zur Verfügung stehenden Mittel. Das von Wolff Schmiegel, Bochum, dem Kongresspräsidenten des 29. Deutschen Krebskongresses, gewählte Kongressmotto „Strukturen verändern - Heilung verbessern" betont die Notwendigkeit dieser Bestrebungen für die Prognoseverbesserung der Patienten mit Tumorerkrankungen in Deutschland - insbesondere angesichts der demographischen Entwicklung und der steigenden Anzahl jährlicher Tumorneuerkrankungen.

\section{Indolente Lymphome}

\section{Bendamustin plus Rituximab}

Die Kombination Bendamustin-Rituximab erwies sich in einer Phase-III-Studie bei Patienten mit follikulären, indolenten und Mantelzell-Lymphomen im Vergleich zu CHOP-Rituximab als überlegen (Rummel MJ et al., 2009, ASH: \#405, oral presentation).

Bei Patienten mit fortgeschrittenen indolenten Non-HodgkinLymphomen gilt CHOP-R (Cyclophosphamid/Doxorubicin/Vincristin/Prednison + Rituximab) als Therapiestandard. In der Phase-IIIStudie der Studiengruppe indolente Lymphome (StiL) erhielten 549 Patienten mit nicht vorbehandelten follikulären, indolenten und Mantelzell-Lymphomen für maximal sechs Zyklen randomisiert entweder Bendamustin $\left(90 \mathrm{mg} / \mathrm{m}^{2} ; \operatorname{Tag} 1+2\right)$ plus Rituximab (375 mg $/ \mathrm{m}^{2}$; Tag 1) alle 28 Tage (B-R) oder Standard-CHOP plus $375 \mathrm{mg} / \mathrm{m}^{2}$ Rituximab alle 21 Tage (CHOP-R).

\section{Längeres progressionsfreies Überleben}

Die Gesamtansprechraten waren mit 92,7\% unter B-R ( $n=260)$ und 91,3\% unter CHOP-R ( $n=253$ ) vergleichbar, die Rate kompletter Remissionen (CR) war unter B-R signifikant höher als unter CHOP-R (39,6\% vs. 30,0\%; $p=0,0262$ ). Beim primären Endpunkt, dem progressionsfreien Überleben, zeigte sich ein signifikanter Vorteil zugunsten von B-R (Abb.). Auch bei der Verträglichkeit wurde der
Vorteil zugunsten der Therapie mit B-R beobachtet: Beispielsweise trat unter CHOP-R eine Leukopenie Grad 3 und 4 signifikant häufiger auf als unter B-R $(38,2 \%$ vs. $12,1 \% ; p<0,0001)$, und Grad-3/4-Neutropenien waren bei den mit CHOP-R behandelten Pati-

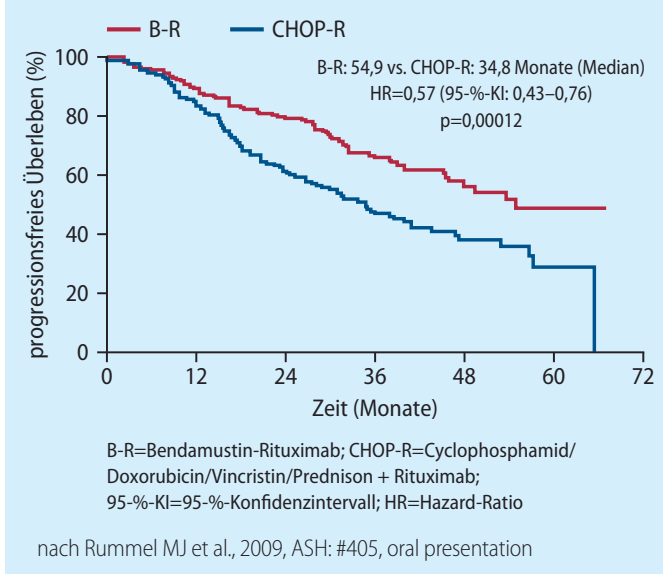

Progressionsfreies Überleben von Patienten mit nicht vorbehandelten follikulären, indolenten und MantelzellLymphomen: Bendamustin-Rituximab versus CHOP-Rituximab (mediane Nachbeobachtungszeit 34 Monate) enten signifikant häufiger (46,5\% vs. 10,75\%; $p<0,0001$ ). „Mit dieser Studie wurden die Voraussetzungen geschaffen, dass CHOP-Rituximab durch Bendamustin-Rituximab als Erstlinientherapie abgelöst werden könnte. Es ist denkbar, dass viele Kollegen das Bendamustin-Regime bevorzugen, weil es effektiver und besser verträglich ist", kommentierte Mathias Rummel, Gießen, die Ergebnisse. $C B$

Quelle: Veranstaltung der Mundipharma GmbH 